\title{
Carotid artery by-pass and head and neck squamous cell carcinoma: Geneva's experience. Should we be afraid of the carotid artery? Nicolas Dulguerov*1,3, Nicolas Murith ${ }^{2}$ and Pavel Dulguerov ${ }^{3}$
}

\author{
Address: ${ }^{1}$ Head and Neck Unit, First Floor East, 250 Euston Road, London NW1 2PG, UK, ${ }^{2}$ Cardio-vascular unit, Geneva University Hospital, \\ HUG, Switzerland and ${ }^{3}$ Head and Neck Unit, Geneva University Hospital, HUG, Switzerland \\ * Corresponding author
}

from Ist Scientific Meeting of the Head and Neck Optical Diagnostics Society London, UK. 14 March 2009

Published: 28 July 2009

Head \& Neck Oncology 2009, I(Suppl I):P32 doi: I0.I I86/I758-3284-I-SI-P32

This abstract is available from: http://www.headandneckoncology.org/content/I/SI/P32

(c) 2009 Dulguerov et al; licensee BioMed Central Ltd.

\section{Purpose}

To determine the survival, the neurologic morbidity, the post-operative surgical complications, and the predictive factors of patients undergoing a radical neck dissection with carotid by-pass for squamous cell carcinoma of the head and neck (SCCHN).

\section{Materials and methods}

A retrospective study of 11 patients operated between 1991 and 2007. Besides survival and recurrence, the type of vascular graft, previous radiation, histological carotid invasion, and major complications were evaluated.

\section{Results}

The 5 -year survival rate was $39 \%$. All deceased patients had a loco-regional recurrence. Salvage surgery and histologic invasion of the carotid artery are negative predictive factors, with an odd-ratio of respectively 6 and 2.5 . The rate of neurological morbity is $9 \%$ and post-operative complications were found in $18 \%$ of patients.

\section{Conclusion}

Radical neck dissection with carotid by-pass achieves a good survival rate considering the advanced disease stage of the patients. Autologous venous or arterial graft should be preferred to avoid neurologic complications. Caution should be observed in case of salvage surgery. 